\title{
A Comparison of Integration Models for Professionals With Different Skills in Healthcare*
}

\author{
Gianluca Lanza \\ University of Parma, Parma, Italy \\ University of Ferrara, Ferrara, Italy \\ Simone Fanelli \\ University of Parma, Parma, Italy
}

\begin{abstract}
Integration between hospital and territory has to be faced as a critical aspect in modern health systems. The evolving of health care demand in Western countries leads the debate on health care system to focus on how to meet the needs of chronic diseases. Creating gatekeeper models for admission to health care facilities presumes a high level of integration between a wide variety of professions that interact in patients' therapy processes. This paper analyzes two models of integration of professionals with different skills: Clinical commissioning groups (CCGs) in United Kingdom health care system and Case della Salute (CdS) in Italian regional health care system of Emilia Romagna. The analysis of the two models highlights critical situations, strong points, and success critical factors in the two models. In particular, the analysis will highlight how the two systems faced the necessity to integrate the different category of professionals that, during the care process of a chronic patient, have to integrate in order to guarantee the continuity of the care process and the most effective response for the patient. This study highlights the methodologies used to share competences and knowledge on which the building of a system with high professional integration is based.
\end{abstract}

Keywords: knowledge management, Case della Salute (CdS), clinical commissioning groups (CCGs), primary care

Knowledge management is a business concept, which includes concerted, coordinated, and deliberate efforts to manage the organization's knowledge through the processes of creating, structuring, disseminating, and applying it to enhance organizational performance and create value (Bose, 2003).

Knowledge management is a process of organizing and distributing an organization's collective wisdom so that the right information gets to the right people at the right time (Robbins, 2003).

The most important goal for the public sector is related to the satisfaction of citizen needs.

Public sector role is going to evolve from a quantity-oriented service to a service focused on quality and in

\footnotetext{
* The results were presented at the 16th European Conference on Knowledge Management in Udine (Italy) on September 3, 2015.

Corresponding author: Gianluca Lanza, M.D., Ph.D. candidate, Department of Public Management, University of Parma, University of Ferrara, Italy; research fields: public administration, public management, government-business relations, public-private partnerships, health care organization, and health policies. E-mail: gianluca.lanza@nemo.unipr.it.

Simone Fanelli, M.D., Ph.D., research fellow, Department of Public Management, University of Parma, Italy; research fields: public administration, public management, public-private partnerships, non-profit organization, health care organization, and health policies. E-mail: simone.fanelli@unipr.it.
} 
a more complex environment (Finger \& Brand, 1999; Pablo, Reay, Dewald, \& Casebeer, 2007; Hartley \& Benington, 2006). This change could be related to an increasing empowerment of citizens that allows them to have a better knowledge about their rights and the standards of service provided. Quality improvement does not necessarily imply less quantity and, according to the economic situation, it means same resources to satisfy needs that are more complex.

At the organizational level, knowledge management provides many benefits for an organization (Cong \& Pandya, 2003):

(1) Improving the organization's performance through increased efficiency, productivity, quality, and innovation;

(2) Organizations that manage knowledge claim higher rates of productivity. By having greater access to their employees' knowledge, organizations make better decisions, streamline processes, reduce re-work, increase innovation, have higher data integrity and greater collaboration. In other words, for public sector, managing knowledge could reduce the cost of operations and improves customer service;

(3) Increasing the financial value of the organization by treating people's knowledge as an asset similar to traditional assets like inventory and capital facilities;

(4) As knowledge transfer is increasingly recognized as a source of value creation, organizations have come to identify knowledge management initiatives as strategic facilitators of competitive advantages;

(5) Evolution of public sector entails an evolution of the people who work inside it and organization models focused on goal-achievement rather than on single phases of a process. It means a new set of tools for increasing cooperation, integration, goal-orientation, knowledge management and diffusion all around the organization. Public sector organizations are not closed or limited to a single building or area; rather they are often located in different places, without hierarchical relation between offices or people that are integrated into the same process. Managing knowledge across public organization is crucial to maintain the value of professionals; lacking in knowledge management, in particular of intangible knowledge, generates loss of information because of organization complexity and of personal knowledge of professionals (Meneguzzo \& Della Piana, 2002). Furthermore, it is important to assess the human factor, who work inside the public sector could be not so willing to share their knowledge with his co-worker. According to Liebowitz and Chen (2003), who work in public sector "keeps knowledge close to their heart as they move through the ranks by the knowledge is power paradigm".

Building knowledge management tools guarantees a better management of knowledge competences in organizations. According to the processes and techniques for managing knowledge in literature, we can identify the following stages (Beckman, 1997):

(1) Identify: determine core competences, recognize strategic capabilities and knowledge domains assess the expertise level for each knowledge domain, and focus on bridging the gap between the existing and needed knowledge;

(2) Capture: attempt to obtain needed knowledge from both inside and outside sources and to formalize and document the obtained knowledge;

(3) Select: assess the value of the captured and formalized knowledge and filter it to obtain knowledge that seems appropriate;

(4) Store: classify the filtered knowledge, get it organized in a standard format, add it to the organizational memory and review and update it periodically; 
(5) Share: classify and retrieve knowledge from organizational memory, and make it available for the knowledge users;

(6) Apply: utilize the knowledge in performing the tasks such as solving problems, making decisions, researching ideas, and learning;

(7) Create: discover new knowledge through a variety of processes such as surveys, best practices, research, pilot studies, and data mining;

(8) Sell: develop and market new knowledge-based products and services.

All the elements above push to the development of integration tools that allow sharing knowledge between different areas or among people and at the same time represent a standard level of action across organization.

\section{Impact on Healthcare}

Healthcare sector, inside public sector, represents a particular context with a higher level of complexity for different reasons:

(1) Informative asymmetry between who provides care and the patient who receives care;

(2) Different professionals have to work together for obtaining a defined goal;

(3) Impossibility to manage most of the care-processes all inside the same facility with the same professionals needing a continuous sharing of information.

Healthcare organizations value is strongly related to the professional knowledge; each professional (physician, nurse, etc.) is part of the organization knowledge as a whole made up by different organs with different roles, acting as a team (Senge, 1994). The importance of professional competences pushes healthcare organizations to build knowledge management tools that allow to diffuse them all around the organization and to make this knowledge as part of organization knowledge. "Core to a knowledge management strategy is to develop a centralized knowledge ‘library’ with various ‘layers’ of information” (Guptill, 2005).

Knowledge in healthcare sector refers to clinical and empirical research. The possibility of defining and formalizing knowledge information through tangible tools allows us to identify it largely as "explicit knowledge" (Polanyi, 1966). According to characteristics of "explicit knowledge", healthcare knowledge is easily stored and retrieved (Wellman, 2009) using tools like documents, databases, etc. (Botha, Kourie, \& Snyman, 2008). An increasing complexity about healthcare sector is related to the importance of continuing update knowledge. The greatest issue about knowledge in healthcare is the accessing and sharing level of the all organization to all information and knowledge. A successful process of sharing knowledge depends on different criteria (Bukowitz \& Williams, 1999):

(1) Articulation: the ability of the user to define what he needs;

(2) Awareness: awareness of the knowledge available. The provider is encouraged to make use of directories, maps, corporate yellow pages, etc.;

(3) Access: access to the knowledge;

(4) Guidance: Knowledge managers are often considered key in the build-up of a knowledge sharing system (Davenport \& Prusak, 1998; Gamble \& Blackwell, 2001). They must help define the areas of expertise of the members of the firm, guide their contributions, assist users, and be responsible for the language used in publications and other communication material. This is so as to avoid an information/knowledge overload;

(5) Completeness: access to both centrally managed and self-published knowledge. The former is often more scrutinized but takes longer to publish and is not as hands-on (and potentially relevant). Self-published 
information on the other hand runs the risk of not being as reliable (Frost, 2014).

The sharing knowledge strategy moves from two different approaches: sharing existing best practice to improve knowledge impact on organization or creating knowledge to increase the quality of processes (Skyrme, 1998). Organization need to manage knowledge is not related only to the collection of single professional competences; a primary role is to diffuse and measure the diffusion all around the organization. According to literature is possible to identify three types of measures to evaluate knowledge implementation program (Guptill, 2005).

(1) Outcome measures: quality and standard performance measurement;

(2) Process measures: activity diffusion;

(3) Satisfaction measures: improvement and satisfaction of care process.

\section{Research Topic}

This paper aims to compare two case studies: Case della Salute (CdS) of Emilia Romagna and clinical commissioning groups (CCGs) of United Kingdom to highlight differences between the two models, in order to: identify if some elements could be identified as critical in their implementation process; understand what elements are useful in a process of sharing competences to increase performances of process.

Inside the different healthcare models all around the world, the authors chose the English one and the Italian one because they are both "Beveridge Model". Beveridge Model structure comes from William Beveridge, the social reformer that designed Britain's National Health Service. In this model, health care is a responsibility of government that provides and finances it through tax payments, just like other welfare activities. In the "Beveridge Model", there is a strong presence of public ownership that controls the major part of hospitals and clinics with the private sector that competes with them through a general accreditation system. These systems show some critical issues according to the evolution of healthcare sector; they are characterized by a low cost per capita among all different healthcare systems and they are facing a challenging issue about relationship between hospital and primary care. Evolution of healthcare needs, increasing of chronic diseases over acute ones have forced government to rethink its goals focusing on keeping patients out of acute facilities offering primary care services in different settings.

CdS and CCG are two different models built to offer primary care services to citizens; differences between CdS and CCG are related to the different healthcare settings chosen by the two countries; to the different timeline of implementation and to the different kind of actor roles into the process.

CCG replaced primary care trusts (PCTs) on April 1, 2013. CCGs are clinically led statutory NHS (National Health System) bodies responsible for the planning and commissioning of healthcare services for their local area. CCGs members include GPs (general practitioners) and other clinicians such as nurses and consultants. They are responsible for about $60 \%$ of the NHS budget and commission most secondary care services such as:

(1) Planned hospital care;

(2) Rehabilitative care;

(3) Urgent and emergency care (including out-of-hours);

(4) Most community health services;

(5) Mental health and learning disability services.

Today in England, there are 211 CCGs, showing a high within variability, according to number of GPs and number of citizens they are responsible for. According to the number of GPs, data from NHS show an 
average of 38 GPs per CCG with a maximum of 127 GPs and a minimum of six GPs; more than 20\% of CCGs are made up by 50 or more GPs and 15\% of CCGs has 20 or less GPs. According to the population they are responsible for, each CCG have 270k of population average with a maximum of $910 \mathrm{k}$ and a minimum of $73 \mathrm{k}$. Table 1 shows the number of citizens served by each CCG.

Table 1

Population Served by Each CCG

\begin{tabular}{ll}
\hline Number of CCG & Population \\
\hline 6 & $<100,000$ \\
76 & $100,000-200,000$ \\
113 & $200,001-500,000$ \\
16 & $>500,000$ \\
\hline
\end{tabular}

Note. Source: elaborations by the authors based on NHS data.

CdS is a reference point for citizens in need of an answer to their health problems. Such as hospitals, these facilities can have different complexity and in relation to the characteristics of the territory and the density of the population, are responsible for (DGR 291/2010, Emilia Romagna):

(1) Providing a single access point to citizens;

(2) Ensuring continuity of care 24/7;

(3) Organizing and coordinating responses to the citizen;

(4) Strengthening integration with the hospital especially in relation to the resignation protected;

(5) Improving the taking over of integrated patients with mental health problems;

(6) Developing prevention programs aimed at the individual, the community, and specific target populations;

(7) Promoting and enhancing the participation of citizens;

(8) Providing continuing education to the operator.

At the time the paper is written, Regione Emilia-Romagna has chosen $120 \mathrm{CdS}, 63$ of 120 are operative yet while other 57 are in process. CdS are divided into three categories according to services there are going to offer: big, medium, and small. All different kinds of CdS contain GP activity. The different services are:

(1) Nurses, midwife, and social assistant (Small CdS);

(2) Consulting clinic (Medium CdS);

(3) Mental health center, public health services, day centers and residence for the various target groups (Big CdS).

Today, the 63 operative CdS are 26 small, 22 medium, and 15 big. CdS respond to different size of population according to their complexity, service offered and orography; according to Emilia-Romagna analysis, $61 \%$ of operative CdS are responsible for 10k-30k citizens. There is no relation between size of CdS and population related; Emilia Romagna Healthcare Authority defines CdS location according to territorial analysis and proximity driver. We can find CdS with different sizes responsible for similar number of citizens:

(1) Small CdS catchment area is between a minimum of 3.503 inhabitants (CdS Berceto, Parma) and a maximum of 54.789 inhabitants (CdS Spallanzani, Reggio Emilia);

(2) Medium CdS catchment area is between a minimum of 5.466 inhabitants (CdS Bedonia, Parma) and a maximum of 79.361 inhabitants (CdS S. Vitale-S. Donato, Bologna); 
(3) Large CdS catchment area is between a minimum of 5.990 inhabitants (CdS Modigliana, Forlì-Cesena) and a maximum of 41.985 inhabitants (CdS City of Parma, Parma).

\section{Methodology and Data}

In order to collect all the information necessary to the analysis, the authors have worked only on paper and official document about CCG, in particular they used official and recognized online sources (e.g., HSCIC, NHS, RCGP, CCGs website). Regarding CdS, this research moved from official legislation and documents from Regione Emilia Romagna integrated with a pair of survey with personnel of Regione Emilia Romagna working on this project.

All data collected have been analyzed qualitatively to extract all the information necessary to compare the different tools used from CCG and CdS.

\section{Analysis}

\section{Clinical Commissioning Groups (CCGs)}

In order to analyze knowledge tools, according to the peculiarity of each CCG, the authors identify one of them, choosing a CCG with a population close to the average. The CCG chosen is NHS Milton Keynes CCG with a population of $266 \mathrm{k}$.

Milton Keynes CCG defines their goals and objectives in a document named "Innovation Strategy".

CCG builds on local strengths to optimize new opportunities for innovation which are driven and supported by research, evidence-based practice, knowledge management, education and training, collaborative working and learning from experience.

The strategy identifies three key roles at board level:

(1) GP Board member education lead;

(2) GP Board member research and evidence lead;

(3) Chief Knowledge Officer...

Milton Keynes CCG defines some strategic goals in order to improve and promote knowledge among professionals:

Promote innovation to support delivery of the QIPP (Quality, Innovation, Productivity, and Prevention) challenge within financial resources by promoting innovation and sharing best practice in commissioning, clinical practice and models of service delivery.

Manage the knowledge base for commissioning and innovation in healthcare and deliver high quality knowledge services that promote innovation in healthcare, and support the application of research evidence to address priorities.

Foster a learning culture and develop collaborative working environment across the CCG that supports learning from experience, innovation, best practice and awareness of research evidence.

Promote research and the use of research to improve healthcare services across Milton Keynes.

Promoting clinical best practice inside CCG reflects the way they choose to share clinical knowledge both to guarantee safe care to the patients and to educate professionals in order to guarantee a high level of competences all around CCG. In particular, Milton Keynes CCG develops some clinical pathways, shared among both professionals and citizens using its official website. Clinical Pathways defined by Milton Keynes CCG are:

(1) Cardiovascular Service; 
(2) Test Endoscopy Service;

(3) Diabetes Services;

(4) Optics;

(5) Stroke and TIA Services;

(6) COPD and Asthma Services;

(7) Referral Management Service;

(8) Primary Care Outpatient Clinics.

\section{Case della Salute (CdS)}

Emilia Romagna Healthcare Authority, during this phase, is going to define all the documents, protocols, and tools that will be applied in each CdS. Structure of CdS reflects an Emilia Romagna Healthcare Authority ongoing project in order to recognize a strong autonomy for each CdS in both clinical and managerial way. Analysis will identify two different tools that manage and develop clinical knowledge inside the CdS: one at regional level and one at CdS level. At the time the authors wrote in Emilia Romagna Region for each patient that seems to be necessary to improve impact of projects through different Health Authorities integrated clinical records are not implemented.

Regional level: Emilia Romagna Healthcare Authority has identified 12 projects: nine mandatory and three suggested.

Mandatory projects related to Primary Care Department and identified by Emilia Romagna Healthcare Authority (DGR 221/2015) are:

(1) Birth path;

(2) Project Hospitalization Risk/Chronicity Profile Risk profile/fragility;

(3) Integrated diabetes management;

(4) Heart failure path;

(5) Management of patients with chronic obstructive pulmonary disease;

(6) Management of people with mental health problems ("Programma Leggieri”);

(7) Cognitive disorders path ("Programma Dementias”);

(8) Pain management;

(9) Management of patients with strong disabilities.

Suggested projects:

(1) Management of patients on oral anticoagulant therapy;

(2) Management of patients with chronic renal failure;

(3) Management of patients with hip fracture.

CdS Level: In each CdS, there are two roles with functional competences in managing clinical knowledge: clinical manager and care manager.

The clinical manager is a physician who coordinates professionals through projects. He works not on hierarchical method but through sharing or creating of processes (including Emilia Romagna Healthcare Authority mandatory projects) and control on their implementation. The clinical manager is also responsible, in addition to Local Health Authority, about physicians' education and evaluation.

The care manager is a nurse specialist with a managerial background. He coordinates, according to the clinical manager, all healthcare professionals inside CdS using projects and process mentioned above through 
their sharing and controlling their implementation. The care manager is responsible, in addition to Local Health Authority, on healthcare professionals' education and evaluation.

\section{Conclusions}

CCG and CdS represent two different experiences of responding to the increasing complexity of healthcare needs. It is important to underline some critical elements useful to understand the differences of two models.

CCG model decentralizes many responsibilities from the health national office; CCGs have to control demand of healthcare needs in a determined area in both clinical and managerial way. They manage budget, define priorities with local authorities, and control hospital demand. Because of this, they have built a more advanced system and CCGs are growing in responsibility and complexity adapting all their managerial tools to this. Focusing on knowledge management tool, they use to develop inside CCG (e.g., clinical pathway, projects, process, etc.). Health Authority helps them to share their knowledge outside CCG borders with IT tools where each CCG can propose their tools (both in clinical and managerial way), so other CCGs can learn, adapt, and/or implement these tools in their context too.

CdS model is very young, almost half of CdS are not operative yet. Young models usually need a strong coordination especially during the first implementation period. According to coordination needs, Regional Health Authority defines CdS tools and strategies, so we can find approximately all the same tools, pathway, managerial role, competences in each typology of CdS.

In conclusion, it seems to be important to underline the key role of knowledge management tools in the transition from hospital-based healthcare context to a multi-center level. Healthcare model history recognizes a primary role to the acute-hospital as principal actor in health supply, but the recent evolution of healthcare needs and the increasing of chronic diseases has created a situation in which acute-hospital are not anymore the best way to meet patients' needs. New models (e.g., CdS and CCG) are less structured than hospitals and they are founded on a new concept of integration and sharing of competences among different kinds of professionals. Knowledge management tools are the main element of a multi-professional organization that needs less hierarchy and more coordination through sharing of different competences and standardization of clinical pathway necessary to guarantee clinical output and outcome.

\section{Research Value}

Research would be a focus on knowledge management tools in healthcare organizations inside the front research of Public Management and Healthcare Management area. Studying managerial choices to control and share knowledge in two particular types of primary care health organization is possible to identify some critical driver to succeed. Two different organizations in different countries allow to find common elements that if repeated elsewhere allow to achieve a defined goal. In reverse, the absence of common elements between the two experiences could be linked to the importance of the context and the cultural characteristics of organizations and professionals.

Analysis is limited only to these two experiences, but it is possible to extend the same model to other organizations in the same countries or in different countries according to the healthcare model adopted or the approach to primary care services.

This paper could represent a first step of CdS growing analysis. The authors expect to develop a new survey process in a year in order to evaluate: How the process is going to be developed, if during development 
process, some elements will change, if CdS will modify their competences and tools inside the healthcare context in Regione Emilia Romagna and if Regione will build some performance indicator on both clinical and managerial outcome.

\section{References}

Beckman, T. (1997). A methodology for knowledge management. International Association of Science and Technology for Development AI and Soft Computing Conference, Banff, Alberta, Canada.

Bose, R. (2003). Knowledge management-enabled health care management systems: Capabilities, infrastructure, and decision-support. Expert System With Applications, 24(1), 59-71.

Botha, A., Kourie, D., \& Snyman, R. (2008). Coping with continuous change in the business environment, knowledge management and knowledge management technology. Oxford: Chandos Publishing Ltd.

Bukowitz, W., \& Williams, R. (1999). The knowledge management field book. London: Financial Times Prentice-Hall.

Cong, X., \& Pandya, K. V. (2003). Issues of knowledge management in the public sector. Electronic Journal of Knowledge Management, 1(2), 25-33.

Davenport, T. H., \& Prusak, L. (1998). Working knowledge: How organizations manage what they know. Harvard: Business Press.

Finger, M., \& Brand, S. (1999). The concept of the learning organization applied to the transformation of the public sector: Conceptual contributions for theory development. In M. Easterby-Smith, L. Araujo, and J. Burgoyne, Organizational learning and the learning organization: Developments in theory and practice. London: Sage.

Frost, A. (2014). A synthesis of knowledge management failure factors. Retrieved from http://www.knowledgemanagement-tools.net

Gamble, P. R., \& Blackwell, J. (2001). Knowledge management: A state of the art guide. London: Kogan Page Publishers.

Guptill, J. (2005). Knowledge management in health care. J Health Care Finance, 31(3), 10-14.

Hartley, J., \& Benington, J. (2006). Copy and paste, or graft and transplant? Knowledge sharing through inter-organizational networks. Public Money \& Management, 26(2), 101-108.

Liebowitz, J., \& Chen, Y. (2003). Knowledge sharing proficiencies: The key to knowledge management. In C. W. Hosapple (Ed.), Handbook on knowledge management 1: Knowledge matter. Berlin: Springer-Verlag.

Meneguzzo, M., \& Della Piana, B. (2002). Knowledge management and public administration. Reconcile the irreconcilable? Azienda Pubblica, 15(4), 489-512.

Pablo, A. L., Reay, T., Dewald, J. R., \& Casebeer, A. L. (2007). Identifying, enabling and managing dynamic capabilities in the public sector. Journal of Management Studies, 44(5), 687-708.

Polanyi, M. (1966). The logic of tacit inference. Philosophy, 41(155), 1-18.

Robbins, S. P. (2003). Organizational behavior (10th ed.). Upper Saddle River, NJ: Prentice-Hall.

Senge, P. M. (1994). The fifth discipline: The art and practice of the learning organization. New York: Currency Doubleday.

Skyrme, D. J. (1998, January). Developing a knowledge strategy. Strategy, 18-19.

Wellman, J. L. (2009). Organizational learning. New York: Palgrave Macmillian. 